\title{
Cognition Based E-education for Advanced Mathematics
}

\author{
Jianhui Liu ${ }^{1}$, and Huichuan Wang ${ }^{1,2 *}$ \\ ${ }^{1}$ Department of Basic Science, Beijing University of Agriculture, Beijing, 102206, China. liujianhui@bua.edu.cn \\ ${ }^{2}$ Beijing Key Laboratory of TCVM, Department of Veterinary Science, College of Animal Science and Technology, Beijing \\ University of Agriculture, Beijing, 102206, China \\ *Correspondence author: Huichuan Wang. Email: *hchwang1@163.com
}

\begin{abstract}
By focusing on the difficulties in teaching and studying advanced mathematics in universities and colleges, this paper present a serious of methods for cognition based e-education. The methods proposed can engage unfocused students and teach complex scientific concepts with a friendly interface. The paper also considers the issues relating to the uneven access to technology of the students, in order to best serve the needs of our student population.
\end{abstract}

Index Terms - cognition based, e-education, advanced mathematics.

\section{Introduction}

With the increasing debates on climate change and the desire for scientific management in various aspects, more and more people have realized the importance of studying scientific subjects. This trend facilitates the increasingly enrolled students in universities and colleges. But how well are the general students equipped to scientifically manage our society? This depends on whether they received a solid science education, and thus it is not surprising that the quality of science and math teaching are once again the subject of intense scrutiny.

However, mathematical education at universities and colleges has always been facing difficulties. For the first reason, the mathematical education in this period tends to go into the "deep ocean" of the origin of advanced math, in order to give students a systematic and innovative point of view. Consequently, the content been taught is much more abstract and tedious for the students. For the second reason, students who accustomed to the step-by-step learning scheme in middle school, feel difficult to catch up the speed of college math education. And once they fail in one class, they often do not understand much more content in the consecutive classes because the concepts in math are more relative. Thirdly, strict advanced math language is so different and so tough from the human characteristics, students who are familiar with ordinary language often feel difficult to understand the college math language, even if they are hard working. On the other side, one university or college math teacher is often in charge of many students comparing to his/her counterpart in middle school, which makes it even more difficult for him/her to give additional lessons for the students lag behind.

Modern pedagogy emphasizes that teachers should give students more free time to investigate the beauty underneath the knowledge. But how to explore the beauty beneath the seemingly dull mathematical knowledge is beyond the scope of many students. Most college students do not know the applied value of abstract mathematical subjects and therefore often have no spirit on them. By contrast, however, there are still some students who spend their leisure time on playing video games despite the fact that their math studies are in difficulties.

These considerations lead to the present study of eeducation strategies in mathematical performance for university students.

\section{Cognition Base Design for E-education in Advanced Mathematics}

Nowadays, the electronic age opens up new horizons for the way we do education. But there are several specific problems related to the e-education in advanced mathematics.

On the one hand, computer and Internet technologies provide us with advanced approaches to teaching and learning. Large screen projection devices installed in classrooms make it possible for computer-aided teaching. But on the other hand, university students, who have difficulties in understanding a mathematical formula written on blackboards in a word-byword modality, do not welcome the way that the formula simply appears as a whole image even if with a slower animation.

Nevertheless, the most suitable way for raising the level of university math education is electronic computational approach. Electronic file copies can really benefit the students studying after school by themselves, with unlimited repeating times. The key issue is how to attract students and how to inspire their spirits. This kind of e-files will not definitely be the records of teachers' speeches. Most students do not like other "teachers" accompanying them after school. They prefer the toy-like friends. Therefore mathematical e-education files should be designed according to their preferences and cognitions.

\section{A. Style Considerations}

We think the displaying interface should be attractively laid out, from the aesthetic to the utilitarian. Only in this way can we compensate the rigorous math properties. From this point of view, colour aspect will be of great importance. It can be used to convey a mood, and to enhance a recall.

Unfortunately, university mathematical teachers typically do not have expertise in the art or pragmatics of colour use. Colours are often selected without considering their physical or psychological effects, and without taking into account design principles and knowledge. In order to enhance the knowledge based design and automatic generation of colour patterns, it is essential to represent the colour design 
knowledge and formalize the colour thinking process in computer systems.

Based on this idea, we previously proposed a formal method to bridge the gap between high-level linguistic colour knowledge and the low-level detailed colour values used in computer system [1]. This is an essential aspect for designing intelligent graphic systems. Next, we will enhance the knowledge based design and gradually realize automatic generation of colour patterns.

\section{B. Dividing Animation Elements to Minimum}

Considering the difficulties of students in understanding the advanced mathematical language, we believe that the document being displayed should be divided into word or math character, each of which having animation in display. In this way, it is possible for most students to work independently and self-paced, before or after ordinary lessons.

Under the condition that displayed math documents have been divided into the minimal elements, computer storage and transmission speed should be considered. This point of view is based on the fact that there are always amount of students who can only use old fashioned computers, mobile-phones etc. In this perspective, computer graphics format should be largely adopted instead of the image format.

\section{Application Considerations}

Under the limited number of lessons and the pressure of difficulties, applied aspects of basic advanced mathematical subjects (calculous, linear algebra and probability) are often neglected during the lecture time. Needless to say, with the basic concept and skills, graduates, in the future, are required to creatively apply advanced mathematics in their majors, especially in applied field. But without knowing the prosperity of application of mathematics, students often lack spirit to challenge the difficulties they are facing with in the basic lessons.

In fact, the more abstract, the wider of the applications. Advanced mathematical subjects have tremendous applications, not just in engineering fields, but also in economical and biological fields. As a consequence of the growing amount of gene expression data, advanced math have entered into the molecular field [2-3]. How to introduce the wonderful applications in various fields to the students? The answer will definitely not be in regular classes, but by eeducation after classes on Internet or on computers. For better engaging unfocused students on the mathematical applications [4-9], we should design elegant animations to describe the complicated documents.

\section{Functional Modules}

With the three designing aspects specified above in section 2, we will propose the modules of e-education in advanced math in this section.

The building blocks should possess the potential ability to motivate the students' learning interests. They can also inspire repetitive practice, which is a key and often valuable aspect of learning. Also, they should have the potential to change the way students learn and can improve the entire learning ecosystem.

There are four basic modules in the e-educational math learning systems: presentation module, imagination module, agent module, and knowledge points' module.

The presentation module is used to introduce the concepts to the students. Instead of ordinary textual screens, we will divide the text and math formula into minimal graphic elements to represent the mathematical concepts. Each graphical element possesses the ability to animate with various styles and speeds, which can be specified by the students interactively.

The imagination module is for students to explore their memories relating to the concepts been introduced in the presentation module. This is an important process to compare the similar concepts.

The agent (module) acts like a person who acts on behalf of the teacher. But comparing to the real teacher, the agent is a cartoon figure who is more lovely and with amusement characteristics. Students can communicate with the agent freely. Thus the agent is more likely a friend to students.

The knowledge points' module is a set of animation clips, each of which displays a definite knowledge point. This module helps students engage in scientific concepts through immersive experiential learning, which often commands greater focus and provides greater rewards for success.

The cooperative working scheme of the four modules is presented in the Fig. 1.

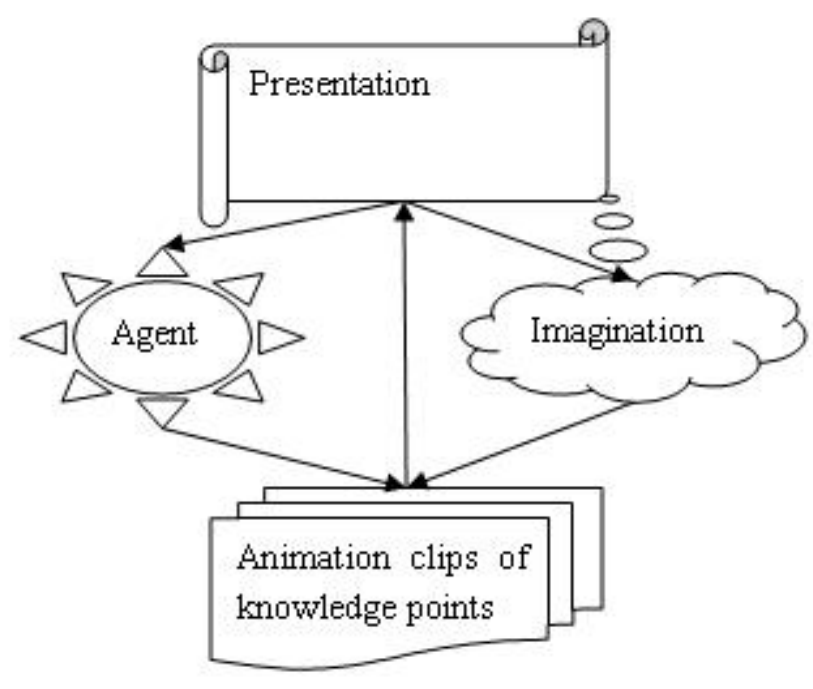

Fig. 1 Basic modules and the working flow.

As illustrated in Fig.1, four modules are closely related. When students find a concept which is confusing in presentation module, he or she will active one of the following modules: 
1) The imagination module: The imagination module will provide the student with some choices concerning the relative knowledge. Each choice activates an animation clip of the corresponding knowledge point. When the clip finishes, if the student understand, the focus will return to the presentation module.

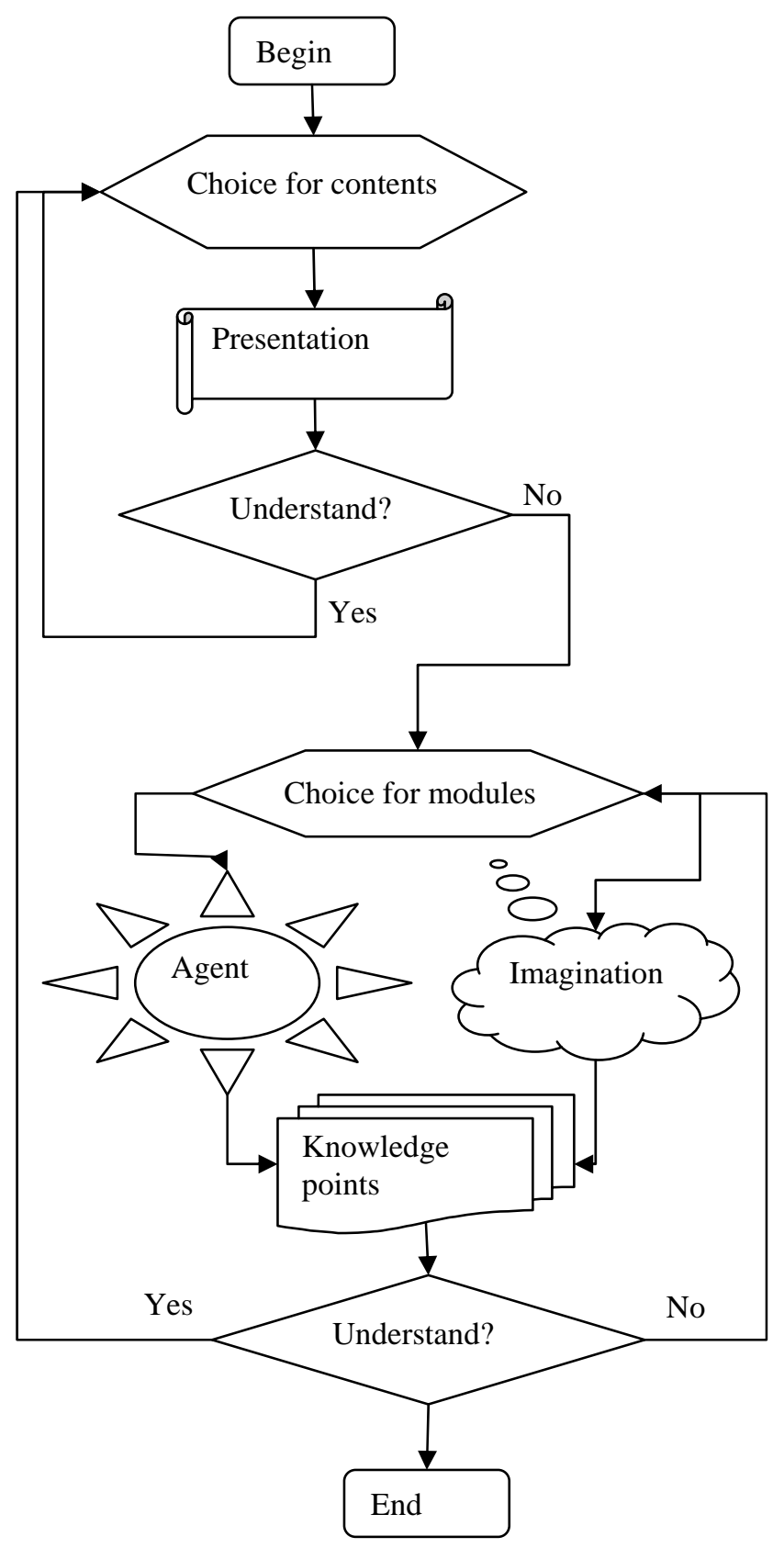

Fig. 2 Flow chart of the process

2) The agent module: The agent module will send an agent which is chosen by the student. The student communicates with the agent, and the agent will guide the student to a relative knowledge point, which also activates an animation clip of the corresponding knowledge point.

These processes can repeat several times until the concept been completely understood by the students. The flow chart of the process is illustrated in Fig.2.

The detailed designing issues for the four modules and their communication scheme is beyond the scope of this paper. But one thing is in certain: the cognition base eeducation will consider to what degree the students can accept the abstract concept. Therefore all the efforts should be around this theme.

\section{References}

[1] J.H. Liu, and S.M. Zhang, "Intermediate fuzzy numbers for color knowledge representation," International Journal of Uncertainty, Fuzziness and Knowledge-Based Systems, vol. 19, no. 5, pp. 879-895, 2011.

[2] U.S. Bhalla, and R. Iyengar, "Emergent properties of networks of biological signaling pathways,” Science, vol. 283 (5400), pp. 381-387, 1999.

[3] J.H. Liu, “Geometric issues for representing protein structure,” Proceedings of The 1st International Conference on Bioinformatics and Biomedical Engineering (ICBBE), pp. 151-154, 2007.

[4] J.H. Liu, "Qualitative orientation reasoning in spatial multi-agent environment," Proceedings of 2005 IEEE/WIC/ACM International Conference(France) on Intelligent Agent technology, pp. 266-272, 2005.

[5] J.H. Liu, and X.F. Zhu, “A coordination model in multi-agent e-commerce environment,” System and Information Sciences Notes, vol. 1, no. 2, pp. 189-194, 2007.

[6] J.H. Liu, and X.L. Du, Linear Algebra- Problem Analysis and Modeling Issues, Beijing, China: China Agriculture Press, 2010. (in Chinese).

[7] B.S. Cheng, and C.C. Wu, "On the calculation of signal transduction ability of signalling transduction pathways in intracellular communication: systematic approach,” Bioinformatics, vol. 28, no. 12, pp. 1604 - 1611, 2012.

[8] Y. Liu, and H. Zhao, “A computational approach for ordering signal transduction pathway components from genomics and proteomics data," BMC Bioinformatics, 5: 158, 2004.

[9] S.M. Gomez, S.H. Lo, and A. Rzhetsky, "Probabilistic prediction of unknown metabolic and signal-transduction networks," Genetics, vol. 159, no. 3, pp. 1291 - 1298, 2001. 